\title{
Regenerative Potential of Dental Pulp
}

\author{
Martin Trope, DMD
}

\begin{abstract}
The regenerative potential of dental pulp, particularly in mature teeth, has been considered extremely limited. However, our improved understanding of pulpal inflammation and repair and improved dental materials and technologies make vital pulp therapy a viable alternative to root canal treatment. This article explores our knowledge in this regard and the future potential of saving or even regenerating the pulp as a routine dental procedure. (J Endod 2008;34:S13-S17)
\end{abstract}

\section{Key Words}

Pulp, regeneration, revascularization, vital

From private practice, Philadelphia, Pennsylvania.

Address requests for reprints to Martin Trope, DMD, 1601 Walnut St, Suite 402, Philadelphia, PA 19102. E-mail address: martin_trope@dentistry.unc.edu.

Conflict of Interest: Martin Trope, DMD, reports no financial interests or potential conflicts of interest. 0099-2399/\$0 - see front matter

Copyright (C) 2008 American Academy of Pediatric Dentistry and American Association of Endodontists.

This article is being published concurrently in Pediatric Dentistry, May/June 2008; Volume 30, Issue 3. The articles are identical. Either citation can be used when citing this article. doi:10.1016/j.joen.2008.04.001

\section{The Importance of Vital Pulp}

Although it is easy to understand the value of a vital pulp when the tooth is immature and underdeveloped, it is also important to understand its value in a fully formed tooth. Endodontic disease is apical periodontitis, and as such, the biologic rationale for endodontics is the prevention or treatment of apical periodontitis. For apical periodontitis to be present, the root canal must contain a necrotic infected pulp (1). Therefore, the vital (noninfected) pulp ensures no apical periodontitis. Thus, maintaining the vital pulp prevents apical periodontitis, and the potential to regenerate an injured or necrotic pulp would be the best root filling possible.

\section{The Unexposed Pulp}

\section{The Pulp's Regenerative Potential}

The inflamed pulp unexposed by caries or trauma always has the potential to be repaired. Although our diagnostic ability to differentiate a vital from a necrotic pulp is good, differentiating between reversibly and irreversibly inflamed pulp remains an educated guess at best (2). We do know, however, that the younger the pulp, the better its repair potential.

\section{The Exposed Pulp}

Treatment of the exposed pulp remains quite controversial, with different approaches endorsed by different dental specialties. Vital therapy (ie, pulp capping, partial or full pulpotomy) on traumatically exposed pulps is very successful (3), whereas vital pulp therapy on the cariously exposed tooth is not nearly as successful (4). The difference in success rates is explained by the status of the pulp at the time of the procedure (Fig. 1). Capping the healthy pulp gives very high success rates, whereas capping the inflamed pulp results in lower and less predictable success (Fig. 2) $(5,6,7)$. On the other hand, with a carious exposure the area and depth of inflammation are very unpredictable, and pulp capping at the superficial exposure site is popular. Thus, it is very likely that we would be capping an inflamed pulp, and more failures (necrotic pulps) would result.

Another extremely important factor in the success of treating a vital exposure is the coronal seal after the pulp capping/pulpotomy (8). Cox et al. (8) showed that the pulp can withstand the toxicity of most dental materials, and that what was previously interpreted as toxicity was, in fact, due to the material not sealing adequately. Therefore, it is considered essential that a well-sealed coronal seal be placed over the vital pulp therapy. This is considered much more important than the material used on the vital pulp.

\section{Approaches to Treatment of Garious Exposure in the Immature Tooth}

\section{Pediatric Dentistry}

Because the young vital pulp has such good potential for repair, it is considered reasonable to perform an indirect or direct pulp cap on a carious exposure as long as a good coronal restoration can be placed (9). The rationale for this approach is that most young pulps can heal as long as the coronal restoration does not allow leakage of additional inflammatory stimulants or microorganisms.

\section{Endodontics}

Pulpal inflammation is usually superficial and is unlikely to extend past the canal orifices, and the healing potential is good if a healthy pulp is treated. Hence, the optimal approach is to perform a full pulpotomy (thus removing the coronally inflamed pulp) and treating the presumably healthy pulp at the canal orifices. When the root canal has 


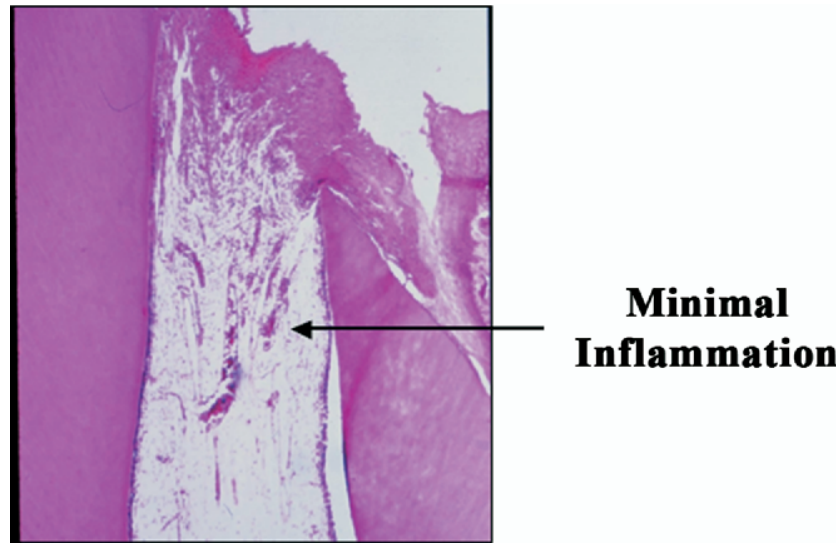

Figure 1. Histologic section of a pulp after a traumatic exposure 24 hours previously. Only about $1 \mathrm{~mm}$ of superficial pulp is inflamed.

developed thick dentinal walls and the apices are closed, a full pulpectomy can be performed (Fig. 3).

\section{Revascularization of Infected Pulp Space Pulp Revascularization}

Revascularization of an immature necrotic tooth has many potential advantages.

It has been shown that under certain conditions revascularization can be achieved in young teeth that have been traumatically avulsed, leaving a necrotic but uninfected pulp. Skoglund et al. (10) demonstrated that in extracted dog teeth, pulpal revascularization started immediately after reimplantation and was completed after approximately 45 days (Fig. 4). It is important to understand the biologic features permitting revascularization in young avulsed teeth, so that we might attempt to reproduce these unique conditions when the pulp space is infected. The immature avulsed tooth has an open apex, short root, and intact but necrotic pulp tissue. Therefore, the new tissue has easy access to the root canal system and a relatively short distance for proliferation to reach the coronal pulp horns. It has been experimentally shown that the apical portion of a pulp might remain vital and proliferate coronally after reimplantation, replacing the necrotized coronal portion of the pulp (11-13). The speed with which the tissue completely revascularizes the pulp space is important because bacteria from the outside are continually attempting to enter the pulp space, and the presence of vital pulpal tissue greatly slows or prevents the bacterial penetration into this tissue compartment. The ischemically necrotic pulp that is unique to an avulsion injury acts as a scaffold into which the new tissue grows, and the fact that the crown is usually intact (rather than carious or with an access cavity) slows bacterial penetration because their only access to the pulp is through cracks (14) or enamel defects. Thus, the race between proliferation of new tissue and infection of the pulp space favors the new tissue.

Revascularization of the pulp space in a necrotic, infected tooth with apical periodontitis was attempted by Nygaard-Ostby and Hjortdal (15) in the 1960 s but was mostly unsuccessful. However, the materials and instruments available $40-50$ years ago were probably not sufficient to create an environment similar to the avulsed tooth, ie, a canal that is free of bacteria, containing a scaffold for new tissue to grow and to be largely resistant to further bacterial penetration. With currently available technologies it could be possible to effectively disinfect an infected pulp, artificially place a scaffold, and then effectively seal the access cavity to resist subsequent infection.

A recent case report by Banchs and Trope (16) has reproduced results in cases reported by others, indicating that it might be possible to replicate the unique circumstances of an avulsed tooth to revascularize the pulp in infected necrotic immature roots (11-13). Our case (Fig. 5) described the treatment of an immature second lower right premolar with radiographic and clinical signs of apical periodontitis with the presence of a sinus tract. The canal was disinfected without mechanical instrumentation but with copious irrigation with 5.25\% sodium hypochlorite and the use of a mixture of ciprofloxacin, metronidazole, and minocycline (17), mixed as described in Fig. 6.

A blood clot was produced to the level of the cementoenamel junction to provide a scaffold for the ingrowth of new tissue followed by a double seal of mineral trioxide aggregate in the cervical area and a bonded resin coronal restoration above it. Clinical and radiographic evidence of healing was observed as early as 22 days. The large radiolucency had disappeared within 2 months, and at the 24-month recall it was obvious that the root walls were thick, and the development of the root below the restoration was similar to the adjacent and contralateral teeth.

The antibacterial effectiveness of the triantibiotic paste reported by Hoshino et al. (17) was confirmed by our group in a dog model with infected immature roots (18). In addition, our group demonstrated in dogs that the potential for revascularization does exist (Fig. 7), and that the blood clot was essential as a scaffold (19). At this point, we are unsure of which factors in the blood clot are important. When these factors are isolated, they can be incorporated into a synthetic scaffold that will be easier to for clinicians to manipulate compared with a blood clot.

The procedure described in this section can be attempted in most cases. If no signs of regeneration are present after 3 months, then more traditional treatment methods can be initiated.

\section{Carious Exposure}
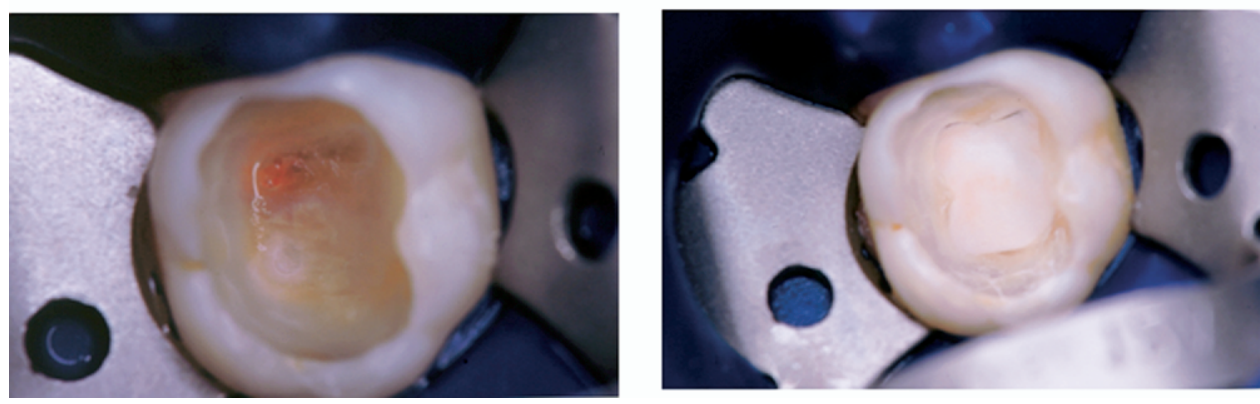

Figure 2. Carious exposure caused by caries. The underlying inflamed pulp is removed, and a partial pulpotomy is performed on the remaining healthy pulp with calcium hydroxide (Courtesy Dr. Francisco Banchs). 


\section{Carious Exposure}
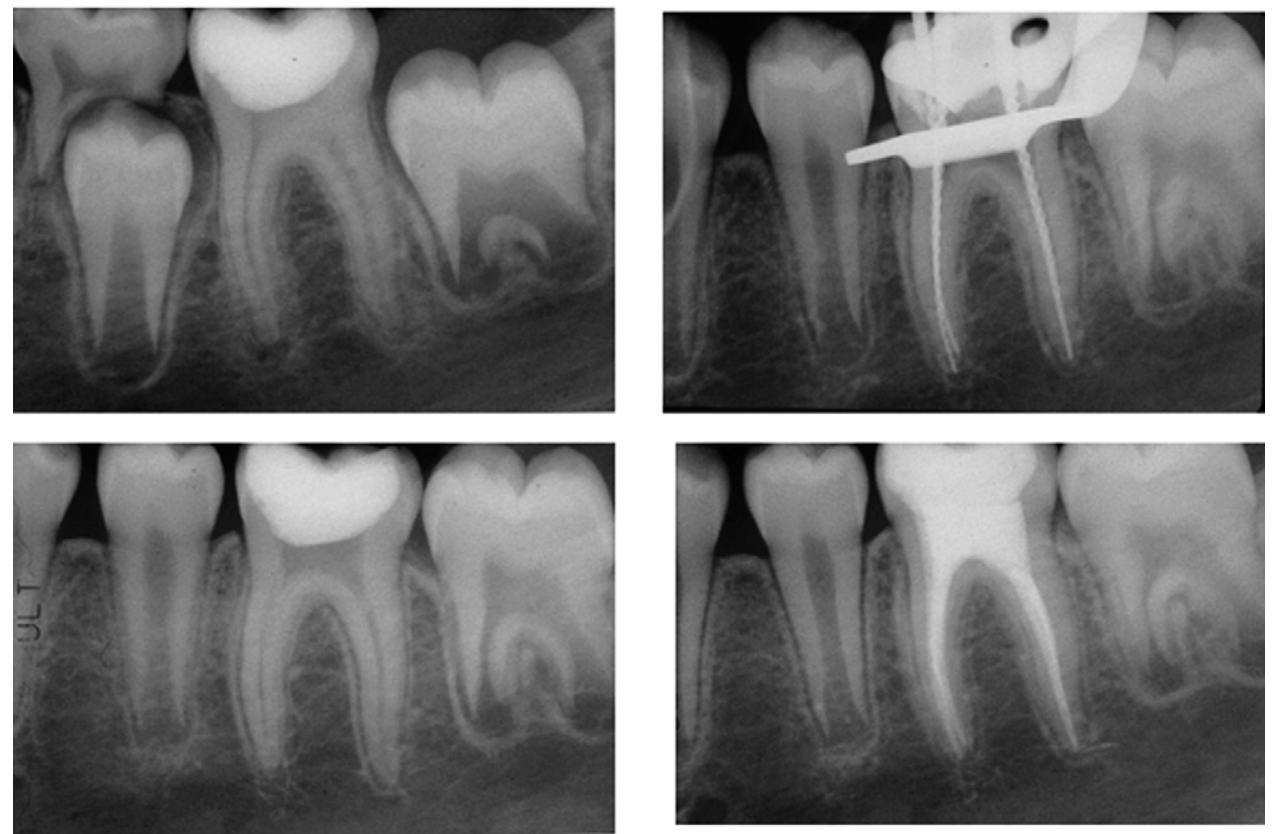

Figure 3. In this case a full pulpotomy was performed (thus removing the coronally inflamed pulp) and treating the presumably healthy pulp at the canal orifices. When the root canal has developed thick dentinal walls and the apices are closed, a full pulpectomy can be done (Courtesy Dr. Francisco Banchs).
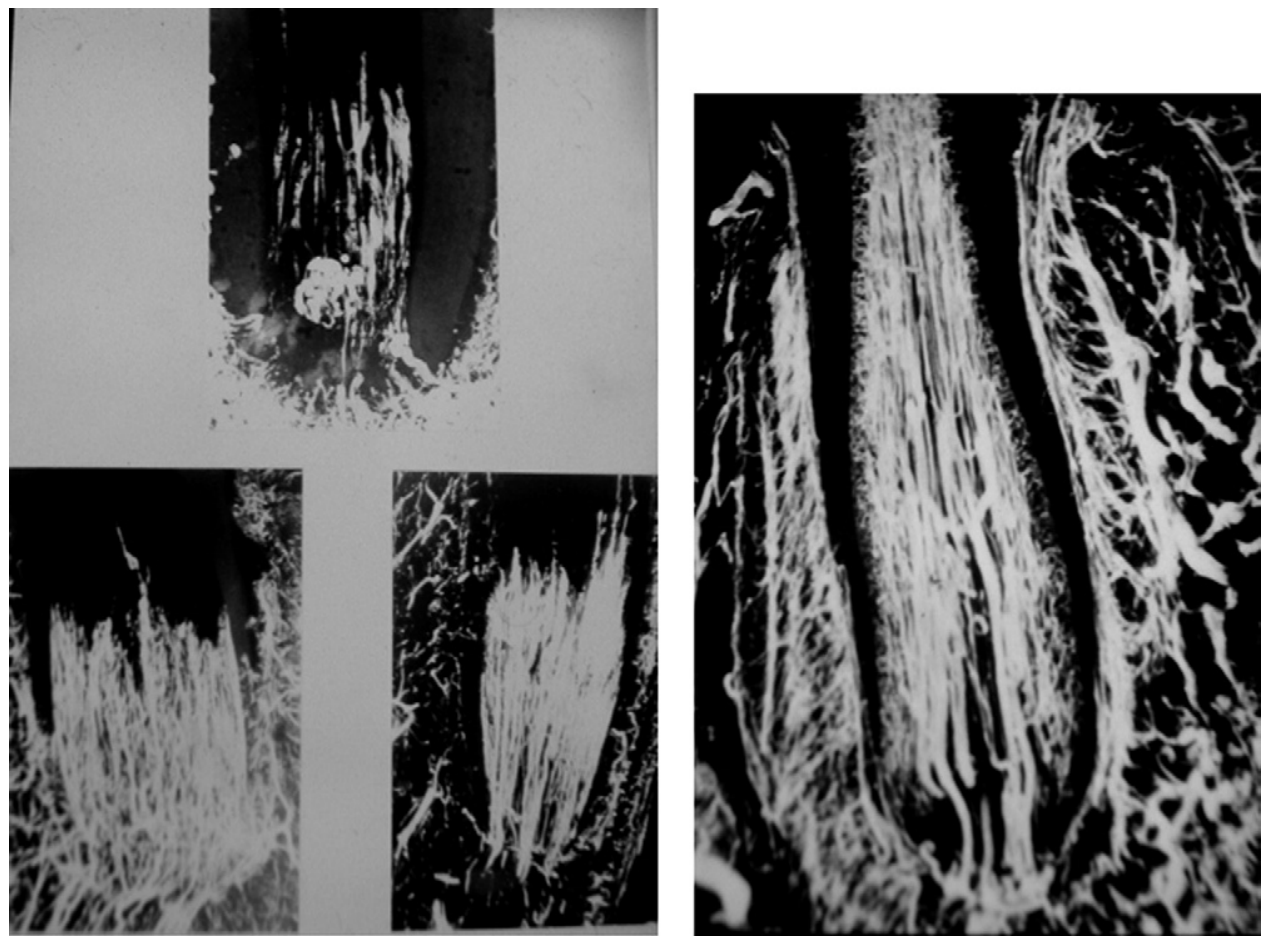

Figure 4. Revascularization of immature dog teeth during period of 45 days. The teeth were extracted and immediately replanted. During the course of 45 days the blood supply moves into the pulp space. 

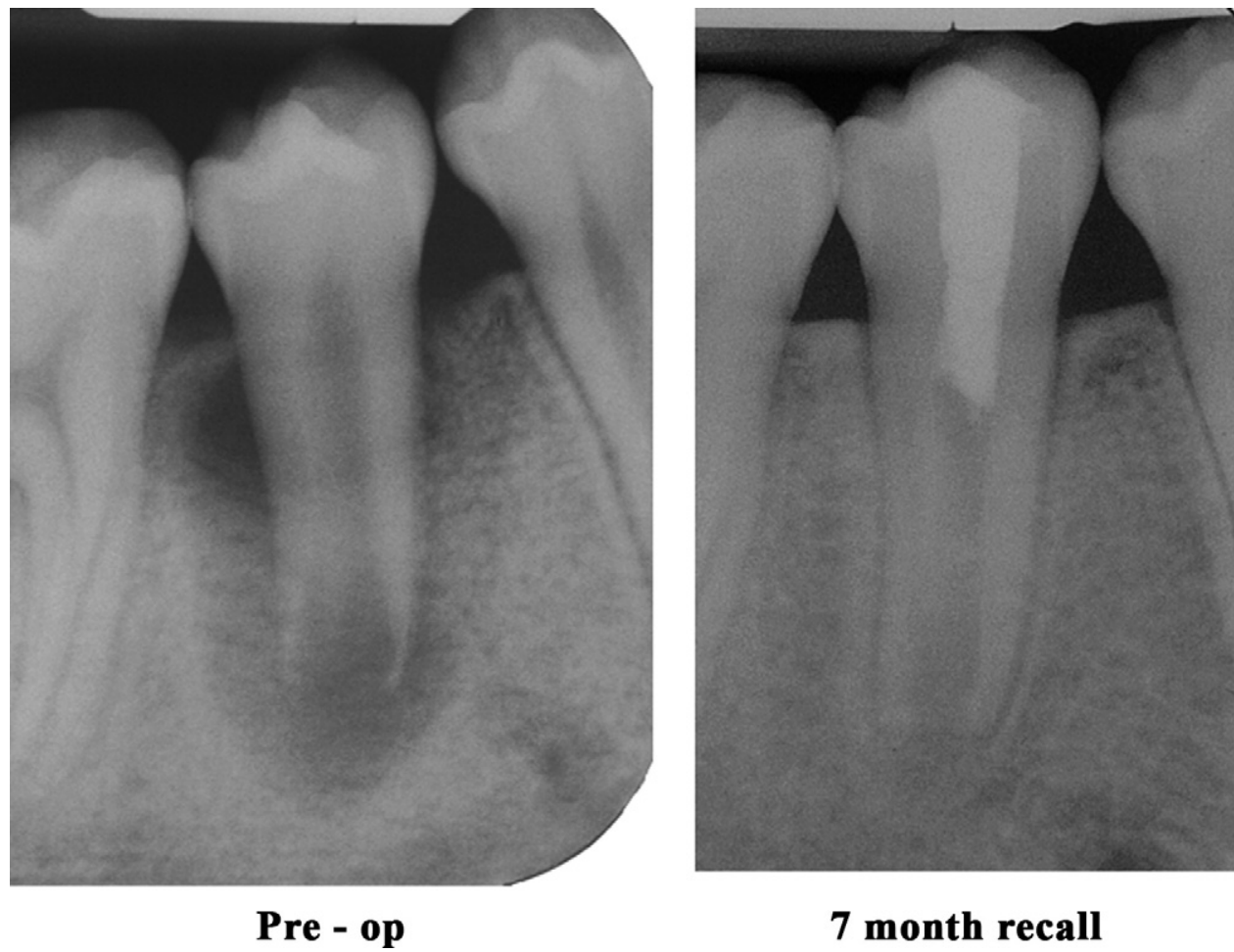

\section{7 month recall}

Figure 5. Immature tooth with a necrotic infected canal with apical periodontitis. The canal is disinfected with copious irrigation with sodium hypochlorite and triantibiotic paste. After 4 weeks the antibiotic is removed, and a blood clot is created in the canal space. The access is filled with a mineral trioxide aggregate base and bonded resin above it. At 7 months the patient is asymptomatic, and the apex shows healing of the apical periodontitis and some closure of the apex. Reproduced with permission from Banchs F, Trope M. Revascularization of immature permanent teeth with apical periodontitis: new treatment protocol? J Endod 2004;30:196-200.

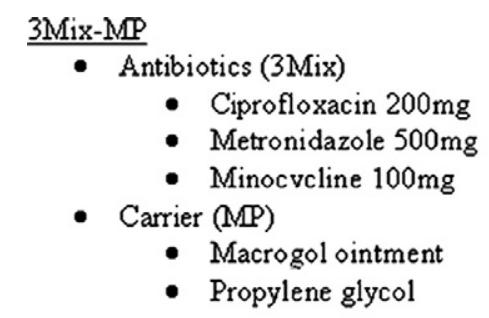

Protocol for preparation

- Antibiotics (3Mix) - be sure to not cross-contaminate

- Remove sugar coating from tablets with surgical blade, crush individually in separate mortars

- Open capsules, crush in individually in separate mortars

- Grind each antibiotic to a fine powder

- Carrier (MP)

- Combine equal amounts of antibiotics (1:1:1) on mixing pad

- Equal amounts of macrogol ointment and propylene glycol (1:1)

- Using clean spatula, mix together on pad

- Result should be opaque

- Separate out small portions of $3 \mathrm{Mix}$ and incorporate into MP using the following:

- $1: 5$ (MP:3Mix) $\rightarrow$ creamy consistency

- $1: 7$ (standard mix) $\rightarrow$ smears easily but does not crumble

- If result is flaky or crumbly, then too much $3 \mathrm{Mix}$ has been incorporated

Storage

- Antibiotics must be kept separately in moisture-tight porcelain containers

- Macrogol ointment and propylene glycol must be stored separately

- Discard if mixture is transparent (evidence of moisture contamination)

Figure 6. Composition and mixing instructions for the triantibiotic paste (adapted from Hoshino et al. (17)). 

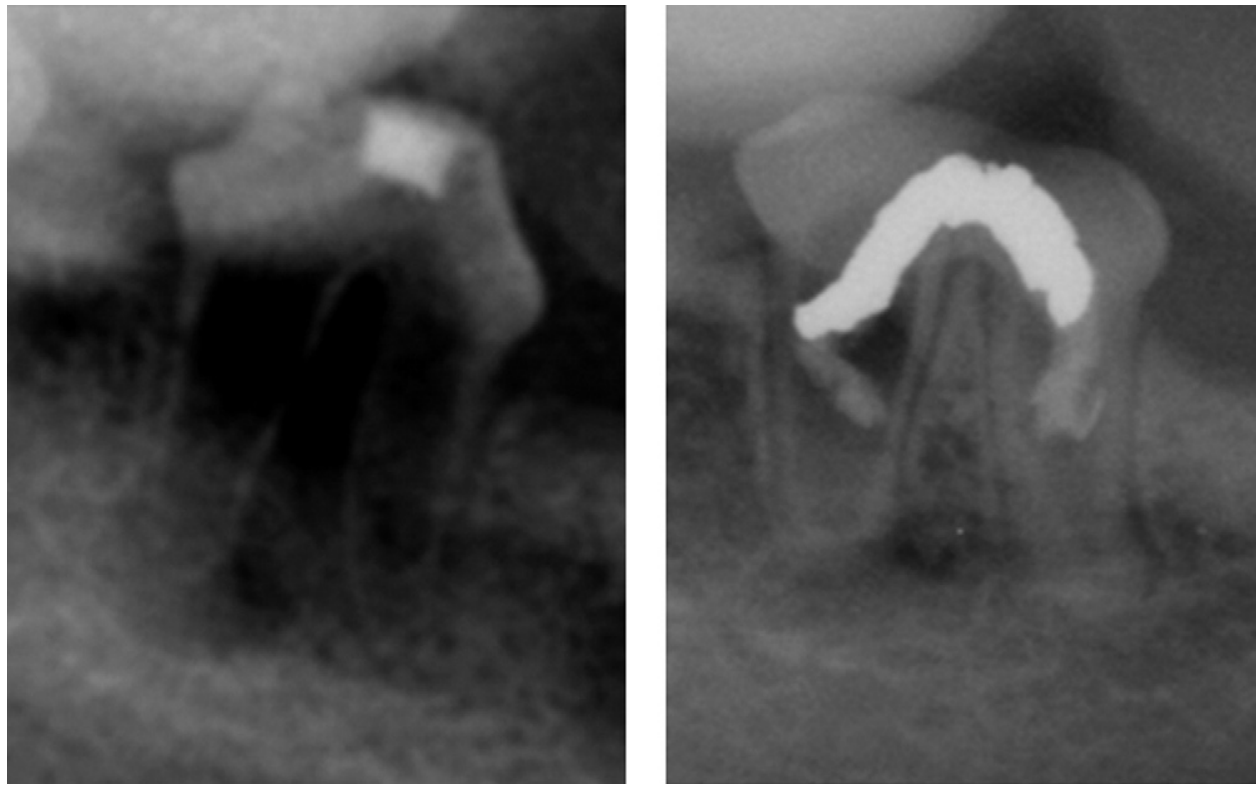

Figure 1. Revascularization of an immature dog tooth with apical periodontitis. The root was artificially infected, producing apical periodontitis. After treatment similar to that described in Fig. 5, revascularization has occurred.

There has been a great deal of discussion as to the correct terminology for what has been called pulp revascularization in this article. Some have used the cases shown as examples of pulp regeneration and the beginning of stem cell technology in endodontics. It is clearly not apexification because not only the apex is closed, but the canal walls are thicker as well. Apexogenesis accomplishes a closed apex and thicker dentinal walls but by definition uses remaining vital root pulp to attain this goal, which is not the case here. Guided tissue regeneration has been used in periodontics and has some merit. However, guided tissue regeneration in periodontics assumes regeneration of periodontal structures, yet this is not the case for pulp. In the present cases, we must distinguish between revascularization and pulp regeneration. Presently, we can only say with certainty that the pulp space has returned to a vital state. On the basis of research in avulsed teeth and a recent study on infected teeth, however, it is more likely that the tissue in the pulp space is similar to a periodontal ligament than to pulp tissue (19). It appears that there is approximately a $30 \%$ chance of pulp tissue reentering the pulp space (20). Future research will need to be performed to stimulate pulp regeneration from the pluripotential cells in the periapical region (21).

\section{References}

1. Bergenholtz G. Micro-organisms from necrotic pulps of traumatized teeth. Odont Revy 1974;25:347-58.

2. Dummer PM, Hicks R, Huws D. Clinical signs and symptoms in pulp disease. Int Endod J 1980;13:27-35.

3. Cvek M. A clinical report on partial pulpotomy and capping with calcium hydroxide in permanent incisors with complicated crown fracture, J Endod 1978;4:232-7.

4. Barthel CR, Rosenkranz B, Leuenberg A, Roulet JF. Pulp capping of carious exposures: treatment outcome after 5 and 10 years: a retrospective study. J Endod 2000;26;525-8.

5. Tronstad L, Mjor IA. Capping of the inflamed pulp. Oral Surg 1972;34:477-83.

6. Mjor IA, Tronstad L. The healing of experimentally induced pulpitis. Oral Surg 1974;38:115-9.
7. Cvek M, Cleaton-Jones PE, Austin JC, Andreasen JO. Pulp reactions to exposure after experimental crown fractures or grinding in adult monkeys, J Endod 1982;8:391-9.

8. Cox CF, Keall HJ, Ostro E, Bergenholtz G. Biocompatibility of surface-sealed denta materials against exposed pulps. Prosthet Dent 1987;57:1-9.

9. Sato T, Hoshino E, Uematsu H, Noda T. In vitro antimicrobial susceptibility to combinations of drugs on bacteria from carious and endodontic lesions of human deciduous teeth. Oral Microbiol Immunol 1993;8:172-6.

10. Skoglund A, Tronstad L, Wallenius K. A microradiographic study of vascular changes in replanted and autotransplanted teeth in young dogs. Oral Surg Oral Med Oral Pathol 1978;1:172-8.

11. Barrett AP, Reade PC. Revascularization of mouse tooth isografts and allografts using autoradiography and carbon-perfusion. Arch Oral Biol 1981;26: 541-5.

12. Rule DC, Winter GB. Root growth and apical repair subsequent to pulpal necrosis in children. Br Dent J 1966;120:586-90.

13. Iwaya SI, Ikawa M, Kubota M. Revascularization of an immature permanent tooth with apical periodontitis and sinus tract. Dent Traumatol 2001;17: 185-7.

14. Love RM. Bacterial penetration of the root canal of intact incisor teeth after a simulated traumatic injury. Endod Dent Traumatol 1996;12:289-93.

15. Nygaard-Ostby B, Hjortdal 0 . Tissue formation in the root canal following pulp removal. Scand J Dent Res 1971:79:333-48.

16. Banchs F, Trope M. Revascularization of immature permanent teeth with apical periodontitis: new treatment protocol? J Endod 2004;30:196-200.

17. Hoshino E, Kurihara-Ando N, Sato I, et al. In-vitro antibacterial susceptibility of bacteria taken from infected root dentine to a mixture of ciprofloxacin, metronidazole and minocycline. Int Endod J 1996;29:125-30.

18. Windley W III, Teixeira F, Levin L, Sigurdsson A, Trope M. Disinfection of immature teeth with a triple antibiotic paste. J Endod 2005;6:439-43.

19. Thibodeau B, Teixeira F, Yamauchi M, Caplan DJ, Trope M. Pulp revascularization of immature dog teeth with apical periodontitis. J Endod 2007;33:680-9.

20. Ritter AL, Ritter AV, Murrah V, Sigurdsson A, Trope M. Pulp revascularization of replanted immature dog teeth after treatment with minocycline and doxycycline assessed by laser Doppler flowmetry, radiography, and histology. Dent Traumatol 2004;20:75-84.

21. Sonoyama W, Liu Y, Yamaza T, et al. Characterization of the apical papilla and its residing stem cells from human immature permanent teeth: a pilot study. J Endod 2008;34:166-71. 